\title{
Phenotypic methods versus PCR-RFLP for the identification of dermatophyte species isolated from patients with dermatophytosis in Egypt
}

\author{
${ }^{* 1}$ Gohar, N. M., ${ }^{1}$ El-Batal, H. M., ${ }^{1}$ Elawady, B. A., and ${ }^{2}$ Samir, N. \\ ${ }^{1}$ Medical Microbiology and Immunology Department \\ ${ }^{2}$ Dermatology Department, Faculty of Medicine, Cairo University, Cairo, Egypt \\ ${ }^{*}$ Correspondence to: nohagohar@yahoo.com
}

\begin{abstract}
:
Background: Dermatophytes are major causative agents of cutaneous fungal infections worldwide. Identification of dermatophyte species is based on macroscopic and microscopic morphology on different culture media. Molecular methods such as PCR-RFLP are rapid, reliable and precise identification methods. This local study aimed to identify the spectrum of dermatophyte species among the studied patients population using different phenotypic and genotypic methods.

Materials and methods: Hair, skin and nail specimens were collected from 135 patients with clinically suspected cases of dermatophytosis. All specimens were subjected to microscopic examination using $\mathrm{KOH}$ and culture on SDA and dermasel agar. Phenotypic identification was done by colony and microscopic morphology, and subculture on malt, PDA, lactrimel and urea agar plates. Molecular identification was done by PCR-RFLP using MvaI.

Results: Out of 135 patients included in the study, $78(57.8 \%)$ were positive by culture for dermatophytes. Five different species were identified, the most commonly isolated species was $M$. canis $(51.3 \%)$ followed by $T$. violaceum $(42.3 \%)$. PCR-RFLP correctly identified the isolated dermatophyte species, producing unique restriction patterns.

Conclusion: Dermatophytosis is common in Egypt where humid hot climate and animal contact play important role in the spread of these fungi. The use of PCR-RFLP directly on clinical specimens rather than its use in the identification of dermatophytes from culture media is recommended.
\end{abstract}

Key words: Dermatophytosis, Dermasel agar, SDA, Sporulation media, PCR-RFLP

Received February 7, 2019; Revised February 22, 2019; Accepted February 23, 2019

Copyright 2019 AJCEM Open Access. This article is licensed and distributed under the terms of the Creative Commons Attrition 4.0 International License (http://creativecommmons.org/licenses/by/4.0), which permits unrestricted use, distribution and reproduction in any medium, provided credit is given to the original author(s) and the source.

\section{Méthodes phénotypiques versus PCR-RFLP pour I'identification d'espèces de dermatophytes isolées chez des patients atteints de dermatophytose en Égypte}

\author{
${ }^{*}{ }^{1}$ Gohar, N. M., ${ }^{1}$ El-Batal, H. M., ${ }^{1}$ Elawady, B. A., and ${ }^{2}$ Samir, N. \\ ${ }^{1}$ Département de microbiologie médicale et immunologie \\ ${ }^{2}$ Département de dermatologie, Faculté de médecine, Université du Caire, Le Caire, Égypte \\ *Correspondance à: nohagohar@yahoo.com
}




\section{Abstrait:}

Contexte: Les dermatophytes sont les principaux agents responsables d'infections fongiques cutanées dans le monde. L'identification des espèces de dermatophytes est basée sur la morphologie macroscopique et microscopique sur différents milieux de culture. Les méthodes moléculaires telles que la PCR-RFLP sont des méthodes d'identification rapides, fiables et précises. Cette étude locale visait à identifier le spectre d'espèces de dermatophytes parmi la population de patients étudiée en utilisant différentes méthodes phénotypiques et génotypiques

Matériels et méthodes: Des échantillons de cheveux, de peau et d'ongles ont été recueillis chez 135 patients présentant des cas suspects de dermatophytose. Tous les échantillons ont été soumis à un examen microscopique utilisant du $\mathrm{KOH}$ et à une culture sur du SDA et de la gélose Dermasel. L'identification phénotypique a été réalisée selon la morphologie des colonies et microscopique, et la sous-culture sur des plaques de malt, de PDA, de lactrimel et d'urée. L'identification moléculaire a été réalisée par PCR-RFLP en utilisant MvaI

Résultats: Sur 135 patients inclus dans l'étude, 78 (57,8\%) étaient positifs en culture pour les dermatophytes. Cinq espèces différentes ont été identifiées. L'espèce la plus communément isolée était M. canis $(51,3 \%)$, suivie de T. violaceum (42,3\%). La PCR-RFLP a correctement identifié les espèces de dermatophytes isolées, en produisant des profils de restriction uniques

Conclusion: la dermatophytose est courante en Égypte, où le climat chaud et humide et le contact avec les animaux jouent un rôle important dans la propagation de ces champignons. L'utilisation de PCR-RFLP directement sur des échantillons cliniques plutôt que son utilisation pour l'identification de dermatophytes à partir de milieux de culture est recommandée.

Mots-clés: Dermatophytose, gélose Dermasel, SDA, milieu de sporulation, PCR-RFLP

\section{Introduction:}

Dermatophytosis is a mycotic infection of skin, hair and nails caused by a unique group of fungi called dermatophytes, which have the ability to infect keratinous tissue. Dermatophytes include 3 important genera; Microsporum, Epidermophyton and Trichophyton (1). Diagnosis and identification of dermatophytes in the laboratory are based on conventional methods such as direct microscopic examination using $\mathrm{KOH}$ and culture-based identification. $\mathrm{KOH}$ mount is rapid, cost-effective and gives a presumptive diagnosis. However it cannot differentiate between different genera and species, and may give false positive or false negative results. Culture is regarded as the gold standard method in identifying dermatophytes but many species are slow growing. Culture on sporulation media is essential to stimulate conidiation, which facilitates species identification (1).

Precise species level identification of dermatophytes is mandatory to know the source of infection whether zoophilic, anthropophilic or geophilic, and to apply proper treatment and control measures (2). Identification of dermatophyte species by traditional methods relies on colony morphology and microscopic features of the colonies on different culture media and on biochemical and physiological tests. However, phenotypic identification is time consuming, requires experienced personnel due to identification overlap between species $(3,4)$.

Genotypic identification using different molecular techniques have been developed and include methods such as nested-PCR, PCR-RFLP, PCR-EIA, Realtime PCR, RAPD-PCR and microarray technology. These methods provide attractive alternative means of identifying fungi including dermatophytes (5). The present study was conducted to determine the prevalence of dermatophyte infections in Egypt, identify different dermatophyte species among the studied patients, and compare the polymerase chain reactionrestriction fragment length polymorphism (PCR-RFLP) analysis with culture-based identification.

\section{Materials and methods:}

The study was approved by the Research and Ethical Committee of 
Medical Microbiology and Immunology Department, Faculty of Medicine, Cairo University. Informed consent was obtained from all patients included in the study.

\section{Demography and clinical history:}

Detailed history obtained from eligible patients included age, sex, occupation, contact with animals, and use of systemic or local antifungal drug in the previous month. Patients who had received systemic or local antifungal therapy in the previous month were excluded from the study

\section{Specimen collection:}

A total of 115 hair, 11 skin and 9 nail specimens were collected from 135 patients with clinically suspected dermatophyte infection, referred to the laboratory of the Cairo Dermatological Hospital "El-Houd El-Marsoud" during the period January and December 2015.

\section{Microscopy and culture of specimen:}

All specimens were subjected to direct microscopic examination using $\mathrm{KOH}$ mount, and cultured on Sabouraud dextrose agar (SDA) (Oxoid, UK) and Dermasel agar supplemented with cycloheximide $(0.4 \mathrm{~g} / \mathrm{L})$ and chloramphenicol $(0.05 \mathrm{~g} / \mathrm{L})$ (Oxoid, UK). Cultures were incubated at 25 to $30^{\circ} \mathrm{C}$ and examined weekly for up to 4 weeks (6). Culture on SDA was used as the gold standard method for the diagnosis of dermatophytosis in the study.

\section{Species identification of dermatophytes using phenotypic methods \\ Fungal growth on culture media was identified by; (i) macroscopic morphology including rate of growth, color of the surface and reverse of the colony, texture of the surface and topography (6); (ii) microscopic morphology using Lactophenol Cotton Blue stain of the Scotch tape preparation and examination under a bright field light microscope for the presence of septate hyphae, chlamydoconidia, microconidia, and/or macroconidia (7); (iii) subculture on 3}

sporulation media; Malt agar (Oxoid, UK), Potato dextrose agar (Oxoid, UK) and Lactrimel agar with subcultures incubated at 25 to $30^{\circ} \mathrm{C}$ and examined weekly for up to 4 weeks by observing colony and microscopic morphology, and (iv) culture on urea agar to differentiate between Trichophyton species (6). Dermatophyte isolates were stored in saline at $-70{ }^{\circ} \mathrm{C}$ for further processing by PCR-RFLP.

\section{Species identification of dermatophytes using PCR-RFLP:}

PCR-RFLP was used as a method for identification of dermatophyte species utilizing MvaI restriction enzyme patterns of PCR-amplified ITS1, 5.8S and ITS2 regions of the ribosomal DNA which revealed unique restriction patterns (1). DNA extraction

DNA extraction was performed for all dermatophytes isolates using i-genomic BYD DNA Mini Kit (Intron Biotechnology, Korea), according to manufacturer's instructions.

Polymerase chain reaction assay Amplification of internal transcribed spacer 1 (ITS1)-5.8S-ITS2 rDNA regions using the ITS1 (forward, 5'-TCC GTA GGT GAA CCT GCG G-3') and ITS4 (reverse, 5'TCC TCC GCT TAT TGA TAT GC-3') primer pairs was done according to previously described protocol (4). The thermal cycler (Biometra T 3000) conditions were as follows; 35 cycles of amplification (denaturation at $95^{\circ} \mathrm{C}$ for $1 \mathrm{~min}$, annealing at $55^{\circ} \mathrm{C}$ for $1 \mathrm{~min}$. and extension at $72^{\circ} \mathrm{C}$ for $2 \mathrm{~min}$ ) ending with a final extension step at $72^{\circ} \mathrm{C}$ for $10 \mathrm{~min}$. Amplified PCR products were run on $2 \%$ agarose gel electrophoresis and visualized by UV transilluminator (BiometraTi). RFLP analysis

The PCR products were digested with the restriction endonuclease enzyme MvaI (Thermo scientific, USA), according to the manufacturer's directions. Restriction fragments were separated by $2 \%$ agarose gel electrophoresis and photographed.

\section{Statistical analysis}

Data were statistically described in 
terms of frequencies (number of cases) and percentages and compared using Chisquare $\left(\chi^{2}\right)$ test. Exact test was used instead when the expected frequency is less than $5, P$ values less than 0.05 was considered statistically significant. All statistical calculations were done using computer program SPSS (Statistical Package for the Social Science; SPSS Inc., Chicago, IL, USA) release 15 for Microsoft Windows.

\section{Results:}

\section{Age and gender distribution of patients:}

The age of the patients ranged between 1 and 42 years, with a mean of $8.71 \pm 6.236$ years. The highest prevalence was observed in patients who were less than 5 years old $(43.7 \%)$ and more males $(63 \%)$ than females were affected.

\section{Diagnosis by clinical presentations:}

Out of the 135 patients; 115 $(85.2 \%)$ were suspected clinically as tinea capitis, $11(8.1 \%)$ as tinea corporis, and 9 $(6.7 \%)$ as tinea unguium. Distribution of dermatophyte species in relation to clinical presentations is shown in Table 1

Table 1: Distribution of dermatophyte species in relation to clinical presentations:

\begin{tabular}{cccc}
\hline Dermatophyte species & Tinea capitis $(\%)$ & Tinea corporis $(\%)$ & Tinea unguium $(\%)$ \\
\hline M. canis $(n=40)$ & $36(50.7)$ & $4(80)$ & 0 \\
T. violaceum $(n=33)$ & $33(46.5)$ & 0 & 0 \\
T. mentagrophytes $(n=1)$ & $1(1.4)$ & 0 & 2 \\
T. verrucosum $(n=1)$ & $1(1.4)$ & 0 & 0 \\
E. floccusum $(n=1)$ & 0 & $1(20)$ & 0 \\
Total $(n=78)$ & $71(100)$ & $5(100)$ & $2(100)$ \\
\hline
\end{tabular}

\section{Diagnosis by direct microscopic examination}

Ninety five out of 135 (70.4\%) specimens were positive on $\mathrm{KOH}$ mount, out of which 67 were positive on culture. Comparison between direct $\mathrm{KOH}$ examination and culture results is illustrated in Table 2, with a statistically significant difference between direct $\mathrm{KOH}$ examination and culture results $(P<$ $0.001)$. The sensitivity, specificity, positive predictive value, negative predictive value and accuracy of direct $\mathrm{KOH}$ examination compared to culture as the gold standard method in diagnosis of dermatophytes were $85.9 \%$, $50.9 \%, 70.5 \%, 72.5 \%$, $71.1 \%$ respectively.

Table 2: Comparison between direct KOH examination and culture results:

\begin{tabular}{ccccc}
\hline \multirow{2}{*}{ Culture } & & $\mathrm{KOH}$ & & Pvalue \\
\cline { 2 - 5 } & & & & \\
Positive & Negative & Total & $<0.001$ \\
Positive & 67 & 11 & 78 & \\
Negative & 28 & 29 & 57 & \\
Total & 95 & 40 & 135 & \\
\hline
\end{tabular}

\section{Diagnosis by culture results:}

Out of 135 patients that were included in the study, $78(57.8 \%)$ were positive by culture for dermatophytes, while $57(42.2 \%)$ were negative. Of 78 culture positive cases, 74 (94.9\%) isolates grew on dermasel agar, 52 (66.7\%) isolates grew on SDA agar and 48 isolates grew on both dermasel agar and SDA. There was a statistically significant difference between culture results on dermasel agar and SDA $(P<0.001)$ (Table 3).

Table 3: Comparison between culture results on dermasel agar and SDA

\begin{tabular}{ccccc}
\hline Culture on & \multicolumn{3}{c}{ Culture on SDA } & Pvalue \\
dermasel agar & & & & \\
\cline { 2 - 5 } & Positive & Negative & Total & $<0.001$ \\
Positive & 48 & 26 & 74 & \\
Negative & 4 & 57 & 61 & \\
Total & 52 & 83 & 135 & \\
\hline
\end{tabular}

Faster rate of growth was observed with dermasel agar; 15 isolates grew in the $1^{\text {st }}$ week on dermasel agar and in the $2^{\text {nd }}$ week on SDA; one isolate grew in the $2^{\text {nd }}$ week on dermasel agar and in the $3^{\text {rd }}$ week on SDA. There was a statistically significant difference between rate of growth on both media $(P=0.002)$. 


\section{History of animal contact:}

Out of 78 culture positive cases, 24 $(30.8 \%)$ had positive history of animal contact (13 patients had history of contact with birds, 7 patients with cats, and 4 patients with dogs).

\section{Species of dermatophytes:}

Out of 78 dermatophyte isolates,

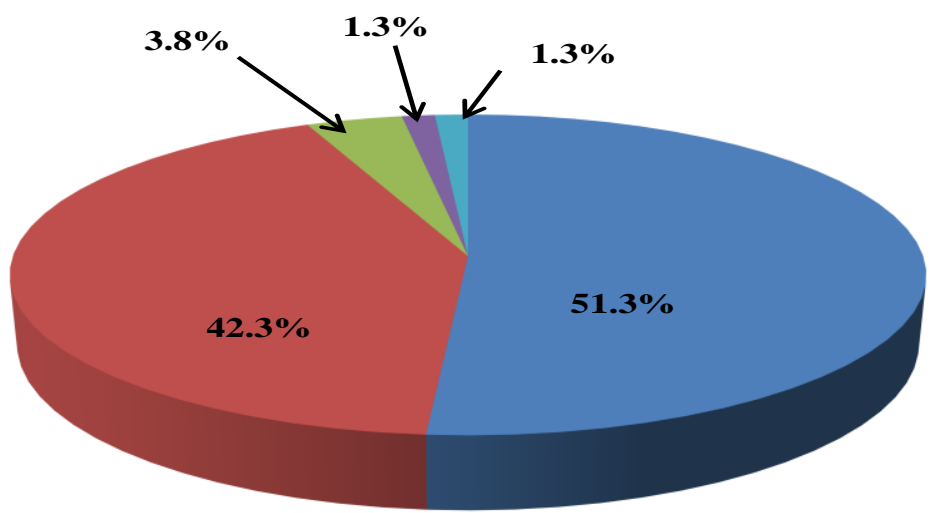

M. canis

T. violaceum

T. mentagrophytes

T. verrucosum

E. floccosum

Figure 1: Distribution of dermatophyte species in 78 patients with dermatophytosis

Microsporium canis isolates were rapid growers, growing during the $1^{\text {st }}$ week or early $2^{\text {nd }}$ week. T. violaceum isolates were slower with growth appearing late in the $1^{\text {st }}$ week or $2^{\text {nd }}$ week. $T$. mentagrophytes and $E$. floccosum isolates also grew during the $2^{\text {nd }}$ week, while $T$. verrucosum isolate was very slow-growing, and grew after 2 weeks of incubation. Growth on sporulation media showed that macroconidia was enhanced in all M. canis isolates, microconidia of $T$. mentagrophytes and macroconidia of $E$. floccusum isolates (Figure 2), while these media failed to stimulate sporulation of all $T$. violaceum isolates and macroconidia of T. mentagrophytes.

One isolate could not be identified due to failure of production of macro and microconidia but was suspected to be $T$. verrucosum based on its colony morphology.

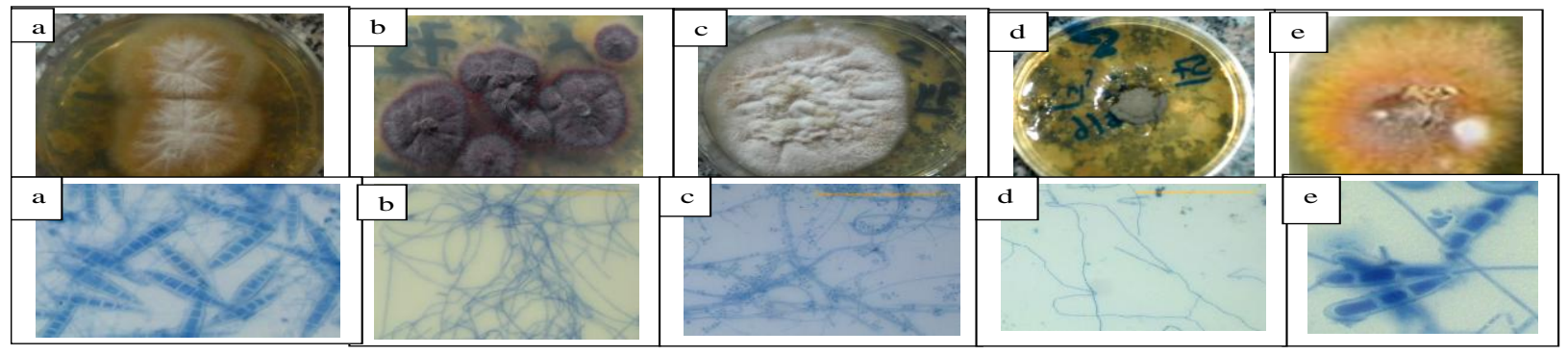

Figure 2: Colony morphology and microscopic morphology of different dermatophyte species on PDA; (a) M. canis, (b) T. violaceum, (c) T. mentagrophytes, (d) T. verrucosum, (e) E. floccosum 
Molecular identification using PCR-RFLP:

PCR-based identification using

ITS1/ITS4 primer set performed on the 78 dermatophyte isolates resulted in amplified products of approximately 690 bp in $T$. violaceum and $T$. mentagrophytes and $740 \mathrm{bp}$ for the $M$. canis and $E$. floccosum. The one dermatophyte isolate which could not be identified phenotypically gave amplified product of approximately 690 bp before digestion with MvaI and the RFLP analysis resulted in four bands typical of $T$. verrucosum. MvaI digestion of the amplified products from each of the five isolated species revealed unique restriction patterns (Figure 3, 4).

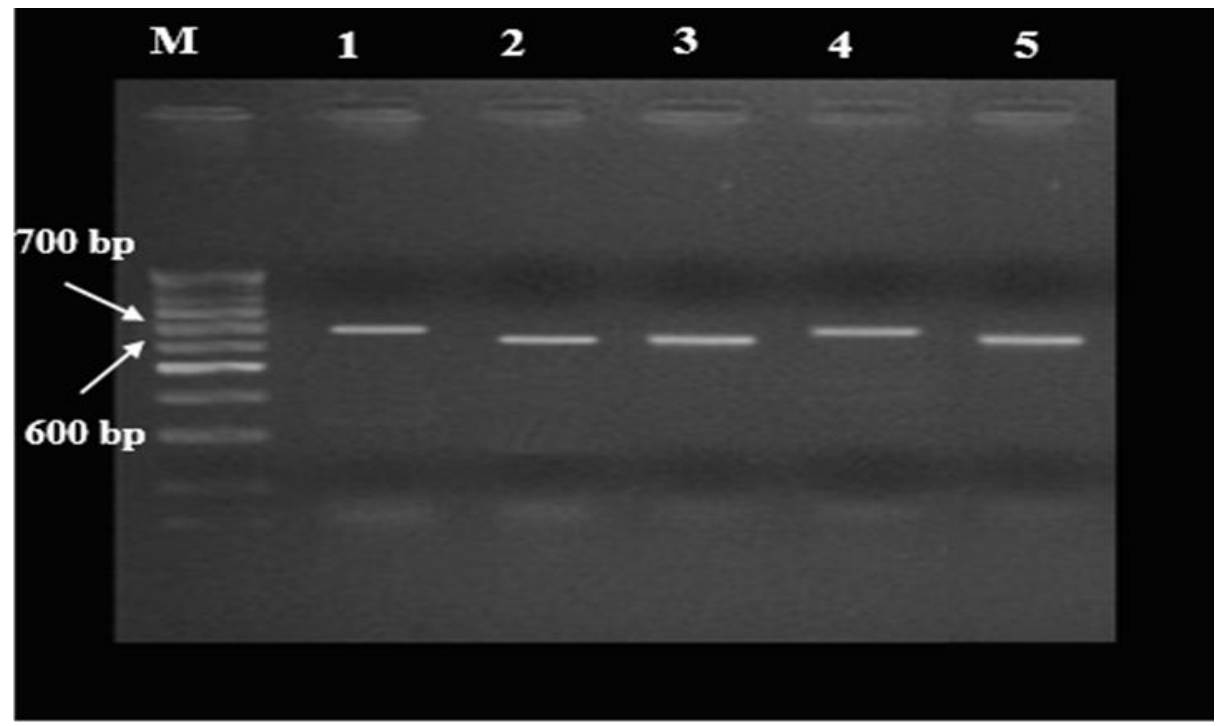

Figure 3: PCR products before digestion with MvaI:

Lanes: Lane M - 100 bp molecular weight marker; Lane 1 - M. canis; Lane 2 - T. violaceum; Lane 3 - T. mentagrophytes; Lane 4 - E. floccosum; Lane 5 - T. verrucosum

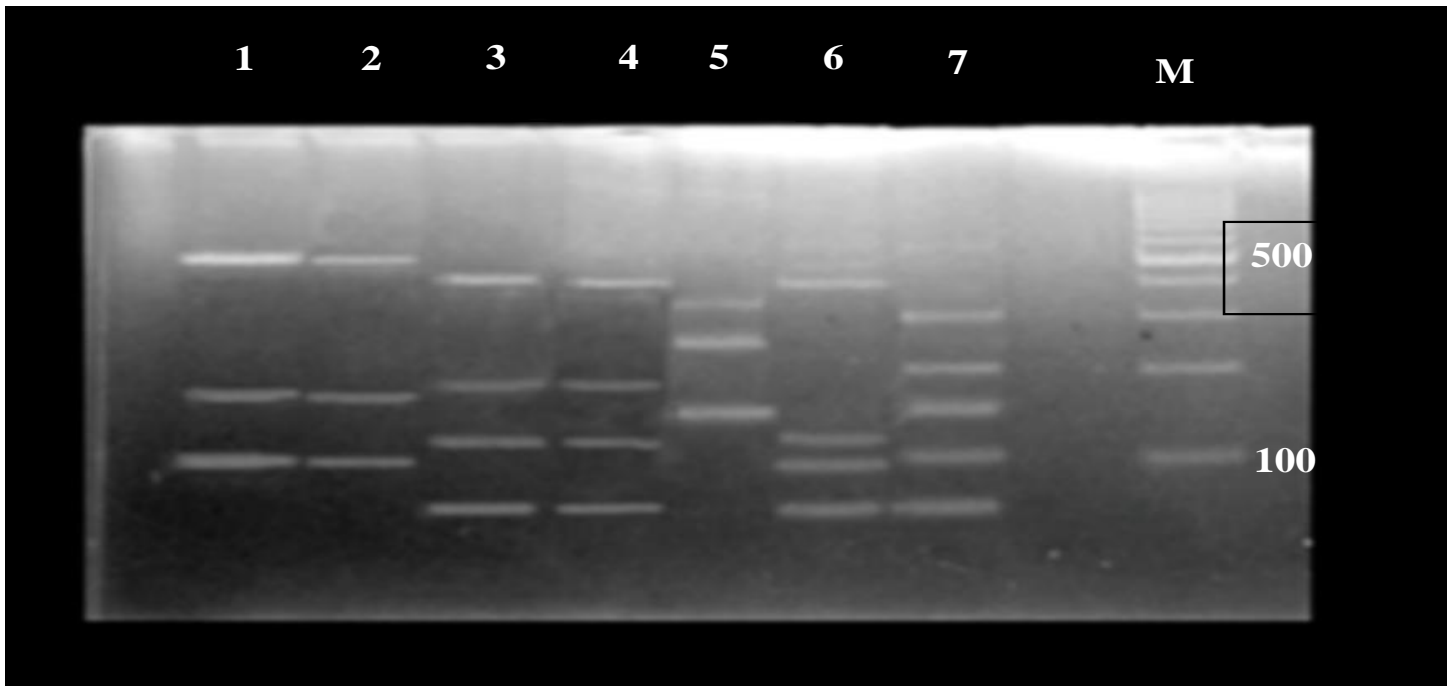

Figure 4: PCR products after digestion with MvaI:

Lanes: Lane M - 100 bp molecular weight marker; Lanes 1 and 2 - M. canis; Lanes 3 and 4 T. violaceum; Lane 5 - E. floccosum; Lane 6 - T. verrucosum; Lane 7 - T. mentagrophytes 


\section{Discussion:}

Dermatophytosis is a major health problem in Egypt. Rapid and precise identification of dermatophytes is essential in order to apply appropriate preventive measures and to direct empirical antifungal therapy (8). The current study was designed to determine the prevalence of dermatophyte infections, identify different dermatophyte species in a group of 135 patients with clinically suspected dermatophyte infections and to compare the PCR-RFLP analysis and culture-based identification methods. The prevalence of dermatophytosis reported in our study was $\mathbf{5 7 . 8 \%}$. Five different dermatophytes species were identified, $M$ canis was the most commonly isolated species $(51.3 \%)$ followed by $T$. violaceum $(42.3 \%)$. The high prevalence of $M$. canis isolated from our study could be attributed to low socioeconomic standard and low hygienic measures of patients and close contact with pets (8).

Two earlier studies from Egypt have reported higher isolation rates for dermatophytosis of $81.5 \%$ and $61.9 \%, M$ canis being the most commonly isolated species in the first study (52.7\%) and $T$. violaceum $(37.3 \%)$ in the second study $(8,9)$. The variation in dermatophytes species isolated in different studies may be explained by the change in climate, environment, geography, lifestyles and occupation (10).

In the present study, the most common clinical presentation was tinea capitis $(85.2 \%)$ followed by tinea corporis $(8.1 \%)$ and tinea unguium (6.7\%). $M$. canis was the most commonly isolated species in both tinea capitis and corporis, while $T$. mentagrophytes was the only species isolated in patients with tinea unguium. Previous studies in Egypt reported that tinea capitis was the most common presentation with rates of $44.5 \%$ and $35 \%$, and $M$. canis and $T$. violaceum were the predominant causative agents $(8,9)$. Tinea capitis was the most common clinical presentation in the Middle East, with the predominance of $M$. canis in Saudi Arabia and Kuwait. T. violaceum was the most commonly isolated species in $83 \%$ of cases in the West Bank of Palestine, $39 \%$ in Iraq and $64 \%$ in Libya (11-15).

In Africa, dermatophytoses are common but are often undetected. The most prevalent clinical form is tinea capitis with $T$. soudanense as the main causative agent in Senegal and Nigeria $(16,17)$. In Ethiopia, a high incidence of tinea capitis mainly caused by $T$. violaceum has been reported (18). In India, tinea corporis was the main clinical presentation and in Japan, tinea pedis was the main clinical form, followed by tinea unguium, $T$. rubrum being the most frequently isolated causal species in both studies $(19,20)$. In Europe, tinea capitis represents about $1 \%$ of dermatophytosis. The predominance of anthropophilic dermatophytes such as $T$. rubrum, M. audouinii, T. violaceum and $T$. tonsurans has been reported (21).

In our study, tinea capitis occurred more commonly in males than females, and $93 \%$ occurred in children below 10 years of age. Male predominance could be explained by the short hairline, increased outdoor physical activities and sweating that favours growth of dermatophytes, and that also increased the chance of exposure to infection in males than females $(9,22)$. Children less than 10 years of age are most susceptible population to tinea capitis due to deficiency in protective fungistatic fatty acids in their scalp as the secretion of sebum starts at puberty, but other predisposing factors reported in literature include close contact with animals and soil, sharing of personal items, overcrowding, poor hygiene measures and low socioeconomic level $(9,22)$.

With respect to the history of animal contact in the present study, $30.8 \%$ of cases had a positive history of animal contact including birds, cats and dogs. The isolated dermatophytes among these patients were $M$. canis followed by $T$. violaceum. This finding agreed to a large extent with that of another study that reported $46 \%$ of cases with positive history of animal contact, and the predominance of $M$. canis, which made the 
authors to conclude that animals play an important role in the transmission of zoophilic dermatophytes, and that they may be the source of anthropophilic dermatophyte infections in human (9). Microscopic examination using $\mathrm{KOH}$ was not adequate for the diagnosis of dermatophytosis in our study. Although the sensitivity $(85.9 \%)$ was high, the specificity $(50.8 \%)$ was low. Another investigator however reported high sensitivity and specificity on direct examination of $88.2 \%$ and $76 \%$ respectively, which might have been attributed to the combined use of $10 \%$ $\mathrm{KOH}$ and $40 \%$ dimethylsulphoxide (DMSO) in that study (8).

Culture is regarded as the gold standard method for primary isolation of dermatophytes. In the present study, $49.6 \%$ of specimens were positive by both $\mathrm{KOH}$ and culture. $\mathrm{KOH}$ could not detect $8.1 \%$ of culture-positive cases, but detected $20.7 \%$ of culture-negative cases, while $21.5 \%$ of cases were negative with both $\mathrm{KOH}$ and culture. Our results are in consonance with other similar studies (23, 24). False negative cases have been attributed to lack of experienced personnel to correctly identify the dermatophytes while false positive cases to early unreported intake of antifungal drugs. In our study, dermasel agar was a useful selective medium for primary isolation of dermatophytes from clinical specimens with $94.9 \%$ of specimens growing on this medium compared to $66.7 \%$ on SDA agar. This agrees with another study (25) but other investigators have reported no significant difference between the isolation rate on both dermasel and SDA (8).

As many species of dermatophytes do not sporulate on SDA medium, it is important to stimulate conidiation for easier identification of species by the use of sporulation media (26). In our study, subculture on sporulation media (Malt agar, PDA and Lactrimel agar) led to enhancement of macroconidia of all $M$. canis and $E$. floccosum isolates as well as microconidia of $T$. mentagrophytes but the macroconidia of $T$. mentagrophytes were not stimulated. The media also failed to stimulate sporulation of all $T$. violaceum and $T$. verrucosum isolates. There was no significant difference between these media regarding sporulation and pigmentation. One study however reported that Lactrimel agar improved macroconidia production after 15 days and was the most useful medium to induce phenotypic characters in daily practice (27).

Identification of dermatophyte species by standard phenotypic methods is time consuming and requires experienced personnel $(3,8)$ necessitating the use of molecular methods several of which have been widely used for identification of dermatophytes. PCR-RFLP is a reliable, easy, simple and precise method for dermatophyte species identification (1). PCR-RFLP assay used in our study enabled the identification of five dermatophyte species and one isolate that phenotypic methods failed to detect was identified as $T$. verrucosum by PCR-RFLP. Several studies have used the PCR-RFLP method for the identification of dermatophyte species with the same single restriction enzyme (MvaI) which we employed in our study. Some investigators have reported that closely related species such as $M$. canis, $M$. ferrugineum, $T$. rubrum and $T$. soudanense gave similar restriction patterns with this enzyme $(1,28)$. Another investigator who performed PCR-RFLP using HaeIII, DdeI and MvaI restriction enzymes reported that the use of DdeI and MvaI yielded unique band profile, however, HaeIII produced similar band profiles and may therefore not be suitable for the identification of dermatophyte species (29).

\section{Conclusion:}

Dermatophytosis is common in our environment where hot humid climate and contact with animals play important role in the spread of these fungi. Tinea capitis was the most common clinical presentation in our study, especially in children below 10 years with male preponderance, and $M$. canis the most 
commonly isolated dermatophyte species. PCR-RFLP correctly identified the isolated dermatophyte species producing unique restriction patterns.

Further studies performed over a longer period of time and covering larger population are recommended to enable better understanding of the epidemiology of dermatophytosis in Egypt. It is also recommended that PCR-RFLP analysis be applied directly on clinical specimens rather than on culture media for identification of dermatophytes.

\section{Conflict of interest:}

interest.

The authors declared no conflict of

\section{Source of funding:}

The study was funded in part by the Faculty of Medicine, Cairo University.

\section{Acknowledgements:}

\author{
Special thanks to "El-Houd El- \\ Marsoud" Hospital for helping in specimen \\ collection
}

\section{References:}

1. Rezaei-Matehkolaei, A., Makimura, K., Shidfar, M. R., et al. Use of single-enzyme PCRrestriction digestion barcode targeting the internal transcribed spacers (ITS rDNA) to identify dermatophyte species. Iran J Publ Health. 2012; 41: 82-94.

2. Aghamirian, M., and Ghiasian, S. Dermatophytoses in outpatients attending the Dermatology Centre of Avicenna Hospital in Qazvin, Iran. Mycoses. 2007; 51: 155-160.

3. Kanbe T. Molecular approaches in the diagnosis of dermatophytosis. Mycopathologia. 2008; 166: 307-317.

4. Shehata, A. S., Mukherjee, P. K., Aboulatta, H. N., et al. Single-step PCR using (GACA) 4 primer: Utility for rapid identification of dermatophyte species and strains. J Clin Microbiol. 2008; 46: 2641-2645.

5. De Baere, T., Summerbell, R., Theelen, B., et al. Evaluation of internal transcribed spacer 2-RFLP analysis for the identification of dermatophytes. J Med Microbiol. 2010; 59: 48-54.

6. Summerbell, R. C. Trichophyton, Microsporum, Epidermophyton and agents of superficial mycoses, Manual of Clinical Microbiology, 2011; $10^{\text {th }}$ ed. Washington ASM Press pp. 1919-1942.
7. St-Germain, G., and Summerbell, R. C. Identifying filamentous fungi: a clinical laboratory handbook. Belmont CA: Star Publishing; 1996.

8. Shalaby, M. F., El-Din, A. N., and El-Hamd, M. A. Isolation, Identification, and In Vitro Antifungal Susceptibility Testing of Dermatophytes from Clinical Samples at Sohag University Hospital in Egypt. Electron Physician. 2016; 8: 2557-2567.

9. Aboueisha, A. M., and El-Mahallawy, H. Public health significance of dermatophytes in Ismailia and Port Said Provinces, Egypt. Med Mycol J. 2013; 54: 123-129.

10. Nweze, E. I., and Okafor, J. I. Prevalence of dermatophytic fungal infections in children: a recent study in Anambra. Mycopathologia. 2005; 160: 239-243.

11. Abanmi, A., Bakheshwain, S., ElKhizzi, N., et al. Characteristics of superficial fungal infections in the Riyadh region of Saudi Arabia. Int J Dermatol. 2008; 47: 229-235.

12. Razzaq, A. A., Sultan, A. O., Basmiah, A. M., et al. Prevalence of tinea capitis in southern Kuwait. Mycoses. 2007; 50: 317-320.

13. Al-Duboon, A. H., Muhsin, T. M., and Al-Rubaiy, K. K. Tinea capitis in Basrah, Iraq. Mycoses. 1999; 42: 331-333.

14. Ali-Shtayeh, M. S., Salameh, A. A. M., AbuGhdeib, S. I., et al. Prevalence of tinea capitis as well as of asymptomatic carriers in school children in Nablusarea (Palestine). Mycoses. 2002; 45: 188-194.

15. Ellabib, M. S., Agaj, M., Khalifa, Z., et al. T. violaceum is the dominant cause of tinea capitis in children in Tripoli, Libya: results of a two year survey. Mycopathologia. 2002; 153: 145-147.

16. Ndiaye, M., Diongue, K., Seck, M. C., et al. Epidemiological profile of Tinea capitis in Dakar (Senegal). A 6 year retrospective study (20082013). J Mycol Med. 2015; 25: 169-176.

17. Ngwogu, A. C., and Otokunefor, T. V. Epidemiology of dermatophytoses in a rural community in Eastern Nigeria and review of literature from Africa. Mycopathologia. 2007; 164: 149-158.

18. Woldeamanuel, Y., Leekassa, R., and Chryssanthou, E. Clinicomycological profile of dermatophytosis in a reference centre for leprosy and dermatological diseases in Addis Ababa. Mycopathologia. 2006; 161: 167-172.

19. Hanumanthappa, S. B., Sarojini, S. B., Shilpashree, S. B., et al. Clinicomycological study of 150 cases of dermatophytosis in a tertiary care hospital in South India. Indian J Dermatol. 2012; 57: 322-323.

20. Sei Y. 2006 Epidemiological survey of dermatomycoses in Japan. Med Mycol J. 2012; 53: 185-192.

21. Ginter-Hanselmayer, G., Weger, W., Ilkit, M., et al. Epidemiology of tinea capitis in Europe: current state and changing patterns. Mycoses. 2007; 50: 6-13.

22. Abd Elmegeed, A. M., Ouf, S. A., Moussa, T. A. A., et al. Dermatophytes and other associated 
fungi in patients attending to some hospitals in Egypt. Br J of Microbiol. 2015; 46: 799-805.

23. Doddamani, P. V., Harshan, K. H., Kanta, R. C., et al. Isolation, Identification and Prevalence of Dermatophytes in Tertiary Care Hospital in Gulbarga District. People's J Sci Res. 2013; 6: 10-13.

24. Yadav, A., Urhekar, A. D., Mane, V., et al. Optimization and Isolation of Dermatophytes from Clinical Samples and In vitro Antifungal Susceptibility Testing By Disc Diffusion Method. J of Microbiol and Biotechnol. 2013; 2: 23202328.

25. Girgis, S. A., Zu El-Fakkar, N. M., Badr, H., et al. Genotypic identification and antifungal susceptibility pattern of dermatophytes isolated from clinical specimens of dermatophytosis in Egyptian patients. Egy Dermatol Online J. 2006; 2: 1-23.
26. Robert, R., and Pihet, M. Conventional Methods for the Diagnosis of Dermatophytosis, Mycopathologia. 2008; 166: 295-306.

27. Ilkit, M., Gümral, R., and Döğen, A. Borelli's lactritmel agar induces conidiation in raremacroconidia producing dermatophytic fungi. Med Mycol. 2012; 50: 735-739.

28. Jackson, C. J., Barton, R. C., and Evans, E. G. Species identification and strain differentiation of dermatophyte fungi by analysis of ribosomalDNA intergenic spacer regions. J Clin Microbiol. 1999; 37: 931-936

29. Elavarashi, E., Kindo, A. J., and Kalyani, J. Optimization of PCR-RFLP Directly from the Skin and Nails in Cases of Dermatophytosis, Targeting the ITS and the $18 \mathrm{~S}$ Ribosomal DNA Regions. J Clin Diagn Res. 2013; 7: 646-651. 